\title{
Longitudinal evaluation of injurious falls and fall prevention strategy use among people with multiple sclerosis
}

\author{
Elizabeth W. Peterson*1, Miho Asano ${ }^{2}$, Marcia L. Finlayson ${ }^{2}$, Michelle H. Cameron ${ }^{3,4}$ \\ ${ }^{1}$ Department of Occupational Therapy, University of Illinois at Chicago, USA \\ ${ }^{2}$ School of Rehabilitation Therapy, Queen's University, Canada \\ ${ }^{3}$ MS Center of Excellence-West, Veterans Administration Portland Health Care System, USA \\ ${ }^{4}$ Department of Neurology, Oregon Health \& Science University, USA
}

Received: September 3, 2015

DOI: $10.5430 /$ jer.v2n2p9
Accepted: November 19, 2015 Online Published: December 3, 2015

URL: http://dx.doi.org/10.5430/jer.v2n2p9

\begin{abstract}
Falls among people with multiple sclerosis (MS) are often injurious. We conducted a prospective cohort study using data collected at baseline, 12 months and 24 months to investigate the prevalence of self-reported injurious falls and trends in fall prevention strategy use among people with MS over this period. Fifty-eight community-dwelling people with MS between the ages of 18 and 50 years, with Expanded Disability Status Scale (EDSS) scores < 6.0, were recruited. Measures included self-reported injurious falls in the past year and scores on the Fall Prevention Strategies Survey (FPSS). A total of 43 subjects completed the study. Prevalence of self-reported injurious falls was $40 \%, 35 \%$, and $16 \%$ respectively at each time point. Seventy-one percent of subjects reporting injurious falls at baseline (12/17) also reported injurious falls at 12 and/or 24 months. Subjects were divided into three subgroups for further analysis: subjects reporting injurious falls at baseline $(\mathrm{N}=17)$; subjects reporting no injurious falls at baseline but subsequent injurious falls $(\mathrm{N}=8)$, and subjects reporting no injurious falls over the 24-months $(\mathrm{N}=18)$. That analysis revealed variations in injurious fall experiences and fall prevention strategy use by subgroup. FPSS scores for each subgroup improved at 24-months compared to baseline. Subgroup analyses yielded insights into sources of variation in injurious fall rates. Findings point to the potential value of using: a) self-reported history of injurious falls to predict future injurious falls; and b) brief interventions to motivate engagement in fall prevention behaviors. Additional studies are needed to test these hypotheses.
\end{abstract}

Key Words: Falls, Multiple sclerosis, Epidemiology, Injuries

\section{INTRODUCTION}

Falls among people with multiple sclerosis (MS) are common and often injurious. ${ }^{[1,2]}$ Identifying people with MS at high risk for injurious falls, providing interventions at an intensity appropriate to risk level, and understanding patterns of prevention behaviors can support cost-effective prevention of fall-related injuries. Previous work undertaken to compare the use of fall prevention strategies by people with MS who do or do not fall revealed that people with MS who fall use more fall prevention strategies than those who do not

\footnotetext{
*Correspondence: Elizabeth W. Peterson; Email: epeterso@uic.edu; Address: Department of Occupational Therapy, University of Illinois at Chicago, 1919 West Taylor Street, MC 811, Chicago, llinois 60612, USA.
} 
fall, and highlighted the need to better understand whether individuals change their strategy use over time. ${ }^{[3]}$ This study examined the prevalence of injurious falls and the use of fall prevention strategies over a 24-month period among a cohort of people with MS.

\section{MeTHODS}

\subsection{Study subjects and methods}

Study subjects and methods have been previously described. ${ }^{[3]}$ Briefly, the data were collected as part of an observational study that included baseline assessment followed by assessment of injurious falls at 12 and 24 months.

\subsection{Data collection and setting}

Subjects were recruited from MS clinics at the Portland Veterans Administration Medical Center (PVAMC), the Oregon Health \& Science University (OHSU), and from surrounding areas via advertising and referral. Institutional review board approval was received from OHSU and PVAMC.

Demographic data and information regarding MS subtype and related disability were collected at baseline. At baseline, 12 and 24 months, subjects also answered the question "Have you suffered any injuries from any falls in the last year?" and completed the Fall Prevention Strategies Survey (FPSS). ${ }^{[4]}$ The FPSS is an 11-item tool developed through
Rasch analysis and used to assess the use of fall prevention strategies among people with MS. Higher FPSS scores (range 0-22) reflect more regular use of more fall prevention strategies. Additionally, the FPSS summarizes the number of falls prevention strategies used. Subjects who reported using a strategy sometimes or regularly were classified as "users" of that strategy; all other subjects were classified as "nonusers". The total number of strategies that participants report using can range from 0 (none) to 11 (all the items).

\subsection{Subjects}

Fifty-eight community-dwelling people with MS between the ages of 18 and 50 years, with Expanded Disability Status Scale (EDSS) scores $\leq 6.0$, were recruited.

\subsection{Statistical methods}

Characteristics of subjects, data on injurious falls, and FPSS data over the study period were analyzed descriptively. SPSS version 22 was used for all analyses.

\section{Results}

Forty-three subjects provided data at baseline, 12 and 24 months (see Table 1). Subjects completing three assessments (median age $=43$, IQR: $36-47$ ) were older than those who missed one or more assessment (median age $=36, \mathrm{IQR}=$ 29-41)

Table 1. Baseline characteristics of subjects and the falls prevention strategies survey data over the 24 month study period by injurious falls status

\begin{tabular}{|c|c|c|c|c|}
\hline & & \multirow{2}{*}{$\begin{array}{l}\text { Subjects who reported a fall } \\
\text { at baseline }(\mathrm{N}=17) \\
\text { Baseline injurious fallers } \\
(\mathrm{N}=17[39.5 \%])\end{array}$} & \multicolumn{2}{|c|}{ Subjects who did not report a fall at baseline $(N=26)$} \\
\hline & & & $\begin{array}{l}\text { Non-injurious fallers } \\
(\mathrm{N}=18[41.9 \%])\end{array}$ & $\begin{array}{l}\text { New injurious fallers } \\
(\mathrm{N}=\mathbf{8}[\mathbf{1 8 . 6 \% ]})\end{array}$ \\
\hline \multicolumn{2}{|l|}{ Mean age at baseline $(S D)$} & $40.6(7.3)$ & $40.6(9.1)$ & $43.7(6.4)$ \\
\hline \multicolumn{2}{|l|}{ Mean expanded disability status } & $2.9(1.4)$ & $2.6(1.7)$ & $2.2(0.8)$ \\
\hline \multicolumn{2}{|l|}{ Female (\%) } & $70.6 \%$ & $72.2 \%$ & $62.5 \%$ \\
\hline \multicolumn{2}{|l|}{ Relapsing remitting MS (\%) } & $94.1 \%$ & $100.0 \%$ & $87.5 \%$ \\
\hline \multirow{4}{*}{$\begin{array}{l}\text { Median falls prevention strategies } \\
\text { Survey total score (IQR) }\end{array}$} & \multicolumn{4}{|l|}{ Time point } \\
\hline & Baseline & $11.0(2.5-16.0)$ & $1.5(0.0-7.25)$ & $4.0(0.25-10.0)$ \\
\hline & 12 month & $10.0(5.0-14.5)$ & $5.0(2.5-8.0)$ & $7.5(4.25-12.75)$ \\
\hline & 24 month & $12.0(5.0-16.0)$ & $6.5(1.0-9.0)$ & $4.5(1.25-7.75)$ \\
\hline \multirow{3}{*}{$\begin{array}{l}\text { Median number of falls prevention } \\
\text { strategies used (IQR) }\end{array}$} & Baseline & $7.0(2.5-9.0)$ & $1.5(0.0-6.0)$ & $3.5(0.25-7.0)$ \\
\hline & 12 month & $7.0(4.5-8.5)$ & $4.5(2.5-6.0)$ & $5.5(3.25-8.0)$ \\
\hline & 24 month & $8.0(4.0-9.0)$ & $5.0(1.0-6.25)$ & $3.5(1.25-6.75)$ \\
\hline
\end{tabular}

Note. SD: Standard deviation; IQR: Interquartile range.

The prevalence of injurious falls for the entire cohort was $40 \%$ (17/43), 35\% (15/43), and 16\% (7/43) respectively at baseline, 12, and 24 months. The 17 subjects who reported injurious falls at baseline were labeled "baseline injurious fallers". Of those 17 subjects, 12 (71\%) also reported injurious falls at 12 and/or 24 months; the 5 remaining subjects 
reported no subsequent injurious falls. Of the 26 subjects reporting no injurious falls at baseline, the 18 (42\%) who reported no injurious falls over the 24-month study period were labeled "non-injurious fallers". The other 8 subjects (19\%) who reported no injurious falls at baseline, but reported injurious falls at $12(\mathrm{~N}=6)$ or 24 months $(\mathrm{N}=2)$ were labeled "new injurious fallers". None of these eight subjects reported falls at both 12 and 24 months.

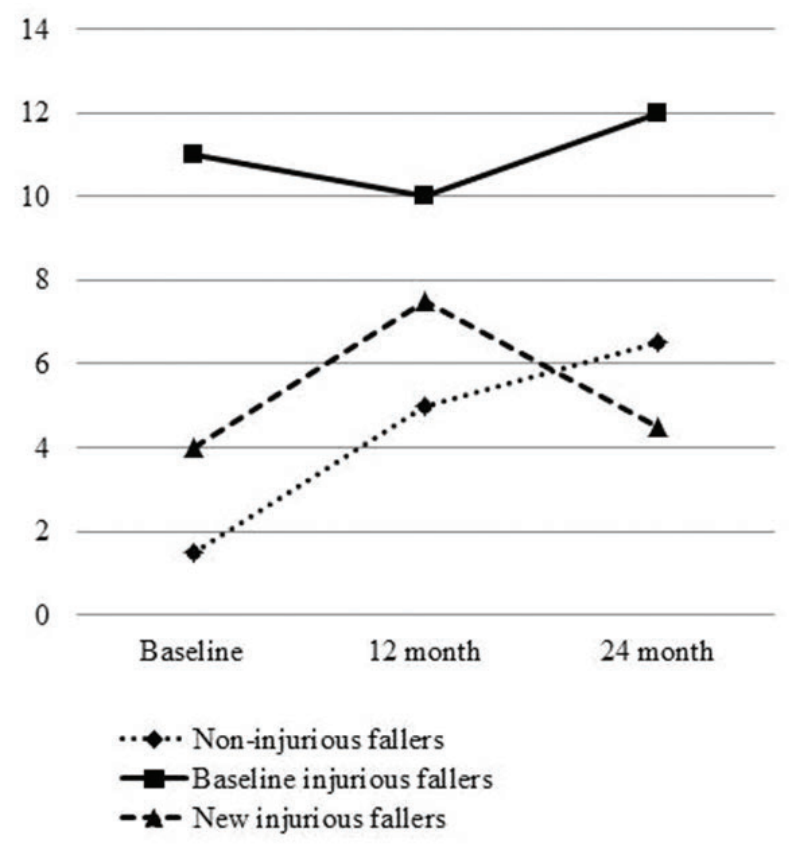

Figure 1. Median falls prevention strategies survey total score over the 24-month study period

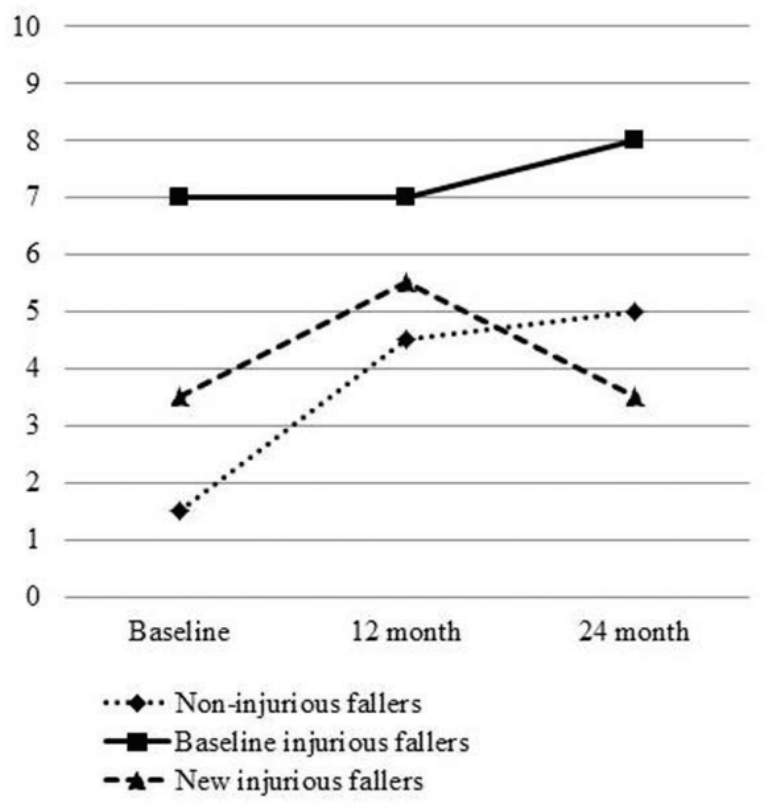

Figure 2. Median number of falls prevention strategies used over the 24-month study period
The patterns of FPSS scores and the number of fall prevention strategies used over the study period varied by group (see Table 1, Figures 1 and 2). Non-injurious fallers showed steady increases in FPSS scores and the number of fall prevention strategies used over the 24 months. Baseline injurious fallers had the highest FPSS scores and used the most fall prevention strategies at all time points. However, baseline injurious fallers showed little change in FPSS scores or number of fall prevention strategies used over time although an upward trend in these subjects' scores and numbers was observed. New injurious fallers showed increases from baseline to 12 months and decreases from 12 to 24 months in both FPSS scores and number of fall prevention strategies used.

\section{Discussion}

Although limited by small sample size and its use of selfreported retrospective data to capture subjects' experience of injurious falls, this study's findings have important implications. Specifically, the variability in the prevalence of injurious falls for the 43 subjects over the 24 months period highlights the value of long-term studies of at least 24 months duration to determine the prevalence of injurious falls for people with MS. Likewise, examining the experience of injurious falls among subjects categorized as "baseline injurious fallers", "non-injurious fallers", and "new injurious fallers" provided insights into sources of variation in injurious fall rates. Findings also suggest the potential value of using history of injurious falls to identify people with MS at risk for future injurious falls. Earlier work indicated that simply asking people with MS if they have fallen in the past year was the best predictor of injurious falls in the subsequent 6 months. ${ }^{[2]}$ The present study builds upon that work by suggesting that self-reported injurious falls may also be a strong predictor of long-term risk for injurious falls. Prospective, longitudinal studies involving larger sample sizes and involving multiple follow-up periods over time are needed to confirm this hypothesis.

While our study was not intended as an intervention study, our findings suggest the potential value of brief interventions to foster adaptive fall prevention behaviors among people with MS. We provided the FPSS to study subjects three times over the 24-month study period, and observed FPSS scores for each subject group that were higher at 24-months compared to baseline. The number of fall prevention strategies used by baseline injurious fallers and non-injurious fallers was also higher at 24-months compared to baseline. It is possible that exposure to the FPSS motivated study subjects to use fall prevention behaviors, and that those behaviors may have contributed to the decline in the prevalence of injurious falls observed. Brief interventions have precedent 
in fall prevention. For example, the U.S. Centers for Disease Control and Prevention created the Stopping Elderly Accidents, Death and Injuries (STEADI) Tool Kit to help health care providers quickly integrate fall prevention education into their practices involving older adults. ${ }^{[5]}$ Because people with MS have unique fall prevention needs related to their symptoms (e.g., fatigue) and typically experience new and progressive symptoms, development and evaluation of brief interventions specially designed for people with MS are indicated.

The promise of interventions designed to reduce fall risk among people with MS has been demonstrated. ${ }^{[6,7]}$ The present study suggests need for future studies to determine cost-effective fall prevention approaches that match programs of varying levels of intensity to target audiences most likely to benefit. Such intervention studies will need to control for, and assess the extent to which observed outcomes are explained by confounding influences (e.g., testing effect, increased engagement in fall prevention activities associated with maturation/living longer with the disease). Qual-

\section{REFERENCES}

[1] Peterson EW, Cho CC, von Koch L, et al. Injurious falls among middle aged and older adults with multiple sclerosis. Arch Phys Med Rehabil. 2008; 89(6): 1031-1037. http://dx.doi.org/10.1016 /j.apmr.2007.10.043

[2] Cameron MH, Thielman E, Mazumder R, et al. Predicting falls in people with multiple sclerosis: Fall history is as accurate as more complex measures. Mult Scler Int. Epub. 2013. http://dx. doi .o $\mathrm{rg} / 10.1155 / 2013 / 496325$

[3] Cameron MH, Asano M, Bourdette D, et al. People with multiple sclerosis use many fall prevention strategies but still fall frequently. Arch Phys Med Rehabil. 2013; 94(8): 1562-1566. http: //dx.doi.org/10.1016/j.apmr.2013.01.021

[4] Finlayson ML, Peterson EW, Fujimoto KA, et al. Rasch validation of the Falls Prevention Strategies Survey. Arch Phys Med Rehabil. itative studies are also needed to understand contextual or activity-related factors that contribute to non-injurious fallers converting to injurious fallers.

\section{Conclusion}

Self-reported history of injurious falls may be a strong predictor of subsequent injurious falls. Brief interventions may promote or sustain engagement in fall prevention behaviors for many people with MS and warrant further investigation.

\section{ACKNOWLEDGEMENTS}

Dr. Cameron's contribution to this study was supported by a Department of Veterans Affairs Rehabilitation Research \& Development Service, Career Development Award (grant no. E7244-W).

Dr. Asano's time on this project was supported by a National Multiple Sclerosis Society mentor-based postdoctoral fellowship (grant no. MB009).

\section{CONFlicts OF INTEREST Disclosure}

The authors have declared no conflicts of interest.
2009; 90(12): 2039-2046. http://dx.doi.org/10.1016/j.apm r.2009.07.013

[5] Centers for Disease Control and Prevention: About CDC's STEADI (Stopping Elderly Accidents, Deaths, \& Injuries) Tool Kit. 2015. Available from: http://www.cdc.gov/homeandrecreationals afety/Falls/steadi/about.html/

[6] Finlayson M, Peterson EW, Cho C. Pilot study of a fall risk management program for middle aged and older adults with MS. NeuroRehabilitation. 2009; 25(2): 107-115. http://dx.doi:10.3233/NR E-2009-0505.

[7] Hugos C, Frankel D, Tompkins SA, et al. Community delivery of a comprehensive fall prevention program improves balance and walking in people with MS: A retrospective observational study. Int J MS Care. Forthcoming. 2015. http://dx.doi.org/10.7224/1 537-2073. 2014-086 